\title{
Incertitudes urbaines et habitantes : l'exemple des hommes célibataires à Arusha (Tanzanie)
}

\section{Mathilde de Blignières}

\section{(2) OpenEdition}

1 Journals

\section{Édition électronique}

URL : https://journals.openedition.org/eastafrica/486

DOI : 10.4000 /eastafrica.486

ISSN : 2790-1076

\section{Éditeur}

IFRA - Institut Français de Recherche en Afrique

\section{Édition imprimée}

Date de publication : 1 juillet 2012

Pagination : 21-28

ISSN : 2071-7245

\section{Référence électronique}

Mathilde de Blignières, «Incertitudes urbaines et habitantes : l'exemple des hommes célibataires à Arusha (Tanzanie) ", Les Cahiers d'Afrique de l'Est / The East African Review [En ligne], 45 | 2012, mis en ligne le 07 mai 2019, consulté le 09 décembre 2021. URL : http://journals.openedition.org/eastafrica/ 486 ; DOl : https://doi.org/10.4000/eastafrica.486 


\title{
Incertitudes urbaines et habitantes : l'exemple des hommes célibataires à Arusha (Tanzanie)
}

\author{
Mathilde de Blignières
}

\section{Introduction}

L'image des villes africaines grossissant au rythme des politiques de développement expansionnistes contemporaines occupe une large part des recherches en sciences sociales dédiées au continent. Dans l'espace est-africain, les principaux centres urbains sont devenus le foyer vivace de nouvelles architectures, tant sur le plan esthétique que sur celui des stratifications sociales. Ces mutations globales, qui alimentent la rhétorique du plus grand, du plus dense et du plus nombreux ne doivent pas pour autant enfermer la ville (est-)africaine au sein d'une définition exclusive qui privilégierait les seuls aspects visibles ou ostensibles de son développement. Car si l'architecture occupe une place de plus en plus importante dans les essais de prise en compte des spécificités du paysage urbain africain, et pour imaginer un nouveau paradigme de la ville africaine du futur, l'infrastructure urbaine fonctionne aussi de manière instable. À Arusha par exemple, principale agglomération du nord de la Tanzanie, dont la population avoisine aujourd'hui les 300000 habitants, la restructuration urbaine qui s'est considérablement accélérée durant ces dix dernières années reste dominée par une forme d'incertitude générale. Celle-ci concerne autant la durée, l'origine que l'objectif des différents projets. L'irrégularité de certaines opérations de restructuration publique, l'opacité qui règne parfois autour de leurs financements, la mise en veille de programmes de démolition ${ }^{1}$, l'irrévocabilité des avis d'expulsion et les promesses arbitraires de relogement font de l'incertitude une réalité incontournable de la vie urbaine moderne. En faisant le constat que la forme construite n'est pas ou n'est plus le résultat d'une planification attentive, d'un aménagement concerté de l'espace urbain, mais la production aléatoire d'un espace de vie pour des groupes humains (de Boeck \& Plissart, 2005 : 233), l'incertitude devient alors partie intégrante du développement de la ville en devenir.

Au-delà des formes extérieures et visibles de la ville, matérielles ou bâties, d'autres espaces intrinsèques plus fluides, relevant de la routine ou des domaines de l'ordinaire, subissent de plein fouet cette incertitude. Des entretiens ont été menés auprès de certains habitants dont le positionnement dans l'espace de la ville semble être

\footnotetext{
1 Ces projets de démolition ont donné lieu par le passé à de nombreuses opérations ostentatoires de rafraîchissement et de marquage de ces mêmes bâtis, semant le trouble auprès des habitants à propos de leur conservation. Le cas est flagrant pour les bâtiments NHC (National Housing Company), datant de la période post-indépendantiste, aujourd'hui en cours de destruction.
} 
en cours de négociation. Il s'agit du profil de quelques hommes célibataires ${ }^{2}$ dont le statut encore peu défini offre un éclairage sur la contrepartie vécue et intime de cette instabilité de façade. Pour ces hommes seuls, devant la difficulté de s'affirmer en tant que médiateurs de leur propre masculinité, urbanité et aussi modernité, à l'échelle du chez-soi comme au sein de l'espace public, nous verrons comment l'incertitude s'exprime au travers de pratiques et autres ressentis résolument inconfortables.

Certains facteurs historiques sont à l'origine du positionnement fragile de ces hommes célibataires dans la ville contemporaine. La violence des déracinements et des migrations de main d'œuvre imposées dès le début du 20ème siècle par le régime colonial allemand est avancé par les historiens de la région comme une première source d'instabilités physiques, pragmatiques et psychiatriques (Sunseri, 2002). Jusqu'à l'indépendance du pays en 1961 et au cours des décennies qui suivirent, les statuts masculins continuèrent d'être mis à mal face à la redéfinition de leurs rôle sociaux, domestiques, dans un contexte politique où l'objectif était de former des foyers sur la base d'une division sexuelle du travail importée, c'est-à-dire devant produire des couples sur le schéma homme salarié/femmes au foyer (Connell, 2000: 205). Enfin la dévaluation des modèles hégémoniques véhiculés par certaines figures sociales du pouvoir et de la réussite (Banégas \& Warnier, 2001 : 5), comme celles du fonctionnaire, du «DG», ou de l'officier qui occupaient auparavant une place centrale dans les imaginaires populaires du succès, acheva d'effriter un positionnement individuel déjà bien affecté par l'explosion du chômage et le développement de la criminalité (Silberschmidt, 2005).

Dans la littérature en sciences sociales dédiée aux masculinités africaines, on assiste depuis les années 2000 à l'introduction du thème de "l'incertitude » comme domaine anthropologique à observer. Le temps de l'incertain, voire de l'invisible donne lieu à de nouveaux discours ainsi que de nouvelles représentations sur la ville, sur l'urbanité et ses conséquences sur le comportement de ses habitants. Dans le cas de la Tanzanie, certaines recherches ont entrepris d'explorer, pour la région d'Arusha, comment les individus coopèrent avec cette idée d'incertitude dans la vie quotidienne, et comment ils développent des perceptions de la «ville-problèmes » d'où sont issus les nouvelles maladies mentales, sexuelles, les déviances religieuses, la multiplication d'actes de sorcellerie, les mauvaises influences, les modes étrangères, les nouvelles déviances, et tout un ensemble de faits qui, pour les personnes vivant à l'extérieur des centres urbains, semblent être interprétés comme autant de conséquences directes de ce qui est autrement qualifié de «moderne» (Haram, 2009). D'autres études mettent en avant l'incertitude économique qui accompagne les réformes néolibérales adoptées par la Tanzanie suite à la libéralisation des marchés dans les années 1980 (Meyer \& Geshiere, 1999). Récemment, des enquêtes menées auprès de clients et gérants de salons de coiffure et autres médiateurs de la culture populaire informelle à Arusha ont illustré comment les transformations des espaces résidentiels et commerciaux affectent les petits

\footnotetext{
${ }^{2}$ Il s'agit d'une quinzaine d'hommes tous originaires de régions rurales du pays et habitant dans le centre-ville d'Arusha ou en périphérie depuis plus de dix ans. Cet article prend place dans le cadre d'un travail de thèse portant sur «les pratiques et les représentations du chez, soi dans l'espace est-africain». Les entretiens ont été conduits entre 2007 et 2009, puis en juin 2011.
} 
commerces de rue, aussi bien dans leurs murs, leur viabilité économique et jusque dans les rêves et les attentes des hommes qui en sont les propriétaires (Weiss, 2009). Les résultats de ces différentes recherches ont permis d'éclairer, dans le cas précis des hommes célibataires, plusieurs défis, parmi lesquels se trouvent la difficulté à prendre place physiquement au sein d'un environnement urbain en refonte, à s'affirmer en tant qu'individu seul et autonome, mais aussi à avancer la part d'indétermination et de subjectivité qu'ils reformulent aussi bien dans leurs rapports au groupe qu'à la tradition. Pour les hommes célibataires, devant les menaces latentes qui pèsent sur la qualité de leur vie quotidienne et face à la fragilité de leur avenir, imposer de nouvelles normes culturelles et genrées (comme celles de la culture hip-hop par exemple) s'affiche parmi les formes de résistance qu'ils déploient. Dans une autre perspective d'analyse, cette foisci genrée, les hommes sont décrits par les femmes comme ne sachant vivre autrement que au jour le jour, dépensant et brûlant leurs ressources dans l'immédiat sans penser à épargner ou anticiper l'avenir (contrairement aux femmes qui doivent penser aux enfants d'abord). La confiance aveugle en l'avenir incertain, imprévisible, semble alors révéler chez certains hommes une capacité à rendre les domaines de l'incertitude familiers.

Pour ces hommes célibataires en quête de repères et de statut urbain, au-delà des manifestations de résistance quotidienne par la démonstration innovante et culturelle de nouvelles présentations de soi, le défi consiste en second lieu à trouver un positionnement parmi les modèles hégémoniques de masculinité qui circulent dans la société tanzanienne. En Tanzanie, plusieurs interprétations «hégémoniques» du masculin sont inspirées des figures du pouvoir et de la réussite qui évoluent au sein des institutions et des espaces publics. Si l'hégémonie prend appui sur la correspondance entre un idéal de culture et le pouvoir institutionnel (Connell, 2005 : 77), la figure du premier président de Tanzanie Julius Kambagare Nyerere (1964-1985) est exemplaire. D'abord parce qu'elle a considérablement marqué l'époque de l'après-indépendance, ensuite parce qu'elle a perduré à travers les mécanismes de production d'une mémoire publique officielle et de ses réappropriations populaires, parfois contestations, dans la Tanzanie post-socialiste (Fouéré, 2009 ; 2011). Sublimées par la Déclaration d'Arusha de 1967, charte fondamentale des politiques de construction de la nation tanzanienne et des fondements du socialisme africain, la politique, tout comme la personnalité de Nyerere, ont longtemps servi de modèles dominants à la fabrication de profils masculins dans la Tanzanie contemporaine. Construites autour de valeurs comme la simplicité et la sobriété, elles ont participé à la définition d'un genre strictement masculin. L'homme issu de ce modèle étatique est un citoyen comme les autres, honnête, intelligent et crédible mais aussi charismatique et autoritaire. Une certaine masculinité de type «bureaucratique » provenant de la création d'une série d'institutions sexuées calquées sur les modèles occidentaux (armée, Etat, bureaucratie, marché de capitaux, marché de main d'œuvre, école, cours de justice, transport) s'est ainsi constituée, donnant lieu à la formation d'élites locales. Bien que les hommes célibataires en particulier disposent a priori d'un potentiel d'indépendance, de mobilité, d'inscription dans la vie active et d'occidentalité, qui sont autant de vecteurs privilégiés d'émancipation vis-à-vis de ces normes genrées, la difficulté pour eux consiste à se positionner entre l'adhésion à des normes et l'envie, très incertaine, de s'en émanciper. 
La construction de sa masculinité et donc de sa légitimité en ville ne nécessite plus aujourd'hui de compter sur sa propre personne mais d'apporter une certaine crédibilité, et si possible de nouvelles disponibilités financières et matérielles. Construire avec assurance sa participation citoyenne et physique dans la ville, prendre conscience également de ce cheminement personnel nécessite alors de dépasser les traumatismes économiques et psychologiques liés aux déracinements, à la fracture des foyers et à la dégradation des modèles masculins, parfois enfouis. Bien que la ville d'aujourd'hui permette, au vu de ses migrations démographiques, une forme de cosmopolitisme, les nouvelles générations de jeunes hommes célibataires, parfois nés sur place, expriment leurs difficultés à s'insérer en tant que tels dans ce cosmopolitisme récent. Alors qu'il existe désormais des moyens émergents de communication virtuelle à domicile (à l'instar de Skype ou de Internet), les difficultés à se faire entendre, se faire reconnaitre, parler de soi et d'autres aspects centraux de toute pratique démocratique semblent omniprésentes pour certains. Pour Achille Mbembe enfin, cette nouvelle urbanité, créole et à bien des égards cosmopolite, accessible au détour de la ville, s'appauvrit cependant de retour chez soi, où l'homme doit faire face à une identité "locale", parfois incomprise ou peu assumée, parfois considérée aussi comme arriérée (Mbembe, 2010 : 210).

\section{Incertitudes (masculines) domestiques et quotidiennes}

C'est en analysant les «bricolages quotidiens » (Welzer-Lang 2000, 2005) faits par les hommes dans l'intimité du foyer et dans la ville que s'éclaircissent alors certains de leurs doutes, de leurs faiblesses et de leurs ajustements, loin des projections intimistes qui sont souvent invoquées à propos de la maison-repli et la maison-protectrice.

Les entretiens menés auprès des hommes célibataires rendent compte en effet de la volonté de se démarquer subjectivement de l'ensemble de ce qui caractérise l'univers domestique au moyen d'une rhétorique particulière. Par exemple les tâches quotidiennes telles que se nourrir, entretenir son linge et sa maison sont souvent décrites comme "futiles» et "ennuyeuses». Le recours à une aide extérieure rémunérée (en général une femme pour laver le linge et un homme pour acheminer l'eau si nécessaire) s'impose comme une alternative. L'habileté à cuisiner seul est soulignée comme une preuve d'autonomie, de débrouillardise et de performance, alors que toutes allusions aux autres pratiques de la chaîne de consommation (comme l'achat de la nourriture, la fréquentation du marché, la préparation et la conservation des aliments) sont en général exclues des discours. Les lieux de vie quotidienne sont en outre couramment disqualifiés, décrits comme "simples », «basiques » et victimes du mauvais état général des infrastructures qui est perçu comme une réelle atteinte à la qualité de la vie. Enfin les contraintes économiques sont omniprésentes dans le discours de ces hommes célibataires. La maison est en général assimilée à un « budget » (incluant les frais de loyer, d'équipement/ameublement, d'eau et d'électricité), qui détermine le choix, l'emplacement et la surface de celle-ci. En définitive, associée à son entretien, la maison de tous les jours paraît réduite à sa valeur la plus " élémentaire », celle d'une structure fonctionnelle, dortoir, vitale, économique, accumulatrice.

Pour ces hommes, cette attitude volontairement détournée des tâches 
domestiques, renforcée par l'expression orale d'un désengagement et le recours à une assistance féminine extérieure, traduit l'inconfort que leur procure le chevauchement avec des espaces socialement définis comme féminins. Devant le risque d'exclusion et de vulnérabilité (à l'égard de la solitude par exemple) qui en découle, la critique du cadre de vie domestique apparaît comme un moyen de justifier leur désengagement à la fois physique et moral des lieux. En outre, les moments d'absence et de présence dans les lieux de vie quotidienne sont mis en scène de façon ostentatoire (par exemple en allumant la télévision ou la radio à plein volume), faisant alterner tour à tour un désir d'habiter les lieux et une volonté d'afficher implicitement sa propre participation à la vie sociale, active, urbaine. Les activités domestiques quotidiennes se résument donc pour ces hommes célibataires à des tâches limitées, répétitives, liées à une forme de maintenance générale qui varie selon le nombre de personnes vivant sous le même toit et la présence ou non de visiteurs.

On observe, face aux mises à distance narratives du contexte domestique, un report de l'engagement personnel sur d'autres aspirations. Guidé par une certaine représentation du confort et de la réussite, l'investissement personnel de ces célibataires urbains semble davantage orienté non plus sur la maison réelle, mais sur la maison rêvée, souvent dessinée dans les imaginaires (ou sous la forme de plans concrets). Utilisée comme support de contestation mais aussi de réflexion, la maison actuelle (et son contexte domestique inclus) est considérée par ces hommes comme une étape passagère vers une installation future. Transitoires puis féminins, ces lieux matérialisent par ailleurs une forme d'ambiguïté sexuelle, car il est un défi pour l'homme célibataire non seulement d'investir «sexuellement» ces lieux, mais aussi de les partager avec le sexe opposé. On en déduit que, pour ces hommes, habiter l'univers domestique et composer avec la solitude au foyer sont potentiellement des sources d'inspiration et de création (aspirations, projections, rêves, fantasmes, désir d'émancipation, projets de vie, etc.), dans lesquelles se dessinent implicitement, au sein d'un processus plus large de progression de la maturité, les contours encore incertains de leur masculinité.

La « construction » des masculinités célibataires prend aussi place dans les lieux publics et urbains. L'observation des hommes en marche dans la ville révèle des habitudes partagées, en dehors ou parallèlement au rythme d'une activité salariale. La plupart ne cuisinent pas pour eux-mêmes et fréquentent les restaurants ou cantines de rue ${ }^{3}$, achètent et lisent la presse à des carrefours particuliers, profitent de l'accès à internet et de la télévision dans certains hôtels, se rendent dans des magasins de pièces détaillées, à l'église le week-end, etc. Leur circulation dans la ville se fait en fonction de lieux-repères qui suggèrent une déambulation «stratégique». Celle-ci leur permet, selon leurs dires, une meilleure intégration dans la ville, tout en poursuivant l'objectif de devenir un « natif », un « résident ${ }^{4}$ ». Pratiquer la ville nécessite en effet de se construire des praxis

\footnotetext{
${ }^{3}$ Il s'agit de restaurants ou stands soit mobiles soit en dur, le plus souvent tenus par des femmes, longtemps appelées en kiswahili «Mama ntilie » (Maman donne moi à manger), devenues aujourd'hui "Mama lishe » (Maman nutrition). Ces appellations réaffirment la répartition sexuelle de certains rôles sociaux dans la société tanzanienne.

${ }^{4}$ En référence au terme swahili de «mwenyeji» qui évoque «celui qui fait partie des nôtres», appellation que souhaite endosser tous primo-arrivants et autres étrangers à la ville au vu d'une intégration à celle-ci.
} 
corporelles, des habitudes, et un rythme synonyme d'occupation de l'espace et du temps. C'est donc le plus souvent par un jeu stratégique et changeant autour de certains profils comme celui de l'intellectuel, du businessman, de l'habitué des lieux, du connaisseur, du dragueur etc., que se façonne sur le bitume l'urbanité de ces hommes. La façon dont les notions de pouvoir qui définissent la nature de l'espace urbain sont puisées à différentes sources de l'imaginaire collectif a, du reste, déjà fait l'objet d'analyses (de Boeck \& Plissart, Ibid.). Dans un mouvement complémentaire, on peut supposer que cette « mise en scène " publique est une façon de contrebalancer les ruptures personnelles qui sont enfouies dans leur espace privé. En effet, outre les différents lieux de socialisation masculine, le domaine de la rue offre la possibilité de se réaliser individuellement au grand jour et en dehors de la solitude imposée par le foyer, lequel reflète entre autres la séparation avec des contextes familiaux, collectifs, ruraux, traditionnels, villageois, corporatistes, communautaires antérieurs. Avec ses rythmes spécifiques, le corps physique détermine ainsi les rythmes du corps social de la ville, et engendre de surcroît des formes spécifiques de vie sociale, en façonnant le sexe de la ville et le noyau d'une partie des «invisibles modalités de l'action urbaine» (de Boeck \& Plissart, op.cit. : 239-240). Le célibat paraît en cela légitimer certaines pratiques de la ville en s'appuyant sur une forme de domination de l'espace public. Ces flâneries, déambulations et autres circuits attribuent en effet à l'homme seul une position particulière d'observateur et acteur des transformations sociales. Cette position se caractérise dans un premier temps par la gestion du temps personnel. Pour ces hommes, la question se pose en effet de savoir si avoir du temps pour soi apparaît comme un statut convenable ou non aux yeux des autres. En général on note que la condition masculine favorise l'adoption d'une position d'entre-deux, d'intermédiaire, en marge, ainsi que l'hésitation, a priori plus aisément que pour les femmes. Par exemple des lieux comme les cantines, les bars ou les hôtels peuvent être plus facilement appréhendés par un homme seul que par une femme seule, les femmes investissant de leur côté (seules ou à plusieurs) des lieux plus cadrés (le marché), institutionnels et à connotation sociale (les hôpitaux, les dispensaires).

Observés sous l'angle de la banalité, la maison comme l'espace urbain sont donc des contextes « ordinaires » dans lesquels se fixent les territoires du masculin, les poches, les lieux d'élaboration et de consolidation de l'identité masculine (Welzer-Lang \& Zaouche-Gaudron, 2011). En se référant au prisme de l'habitat, l'habitant et l'habiter, mis en place par la sociologie de l'habitat ${ }^{5}$, la maison agit comme un premier repère qui structure le temps - en faisant le lien entre le présent, le passé et l'avenir - et l'espace. C'est là qu'émergent les premières déclinaisons de l'identité masculine, comme acteur, habitant et homme au foyer. L'analyse de ces hommes dans la maison et l'espace domestique pose d'autre part la question de savoir si l'individu produit un espace matériel non pas comme un objet détaché de lui mais bien comme une modalité de sa propre existence, ce à quoi la réponse semble résider dans la représentation et la maturation d'un projet de maison à soi. Le gain d'autonomie et de maturité qui se dégage en échange de ces aspirations/réflexions tournées vers l'avenir s'affiche alors comme une forme d'alternative à une masculinité en cours de construction et aussi mise à

\footnotetext{
${ }^{5}$ Voir les travaux de Philippe Bonnin sur la «domus » qui regroupe, selon l'auteur, l'espace de vie, l'espace vécu et l'espace projeté (Bonnin \& Villannova, 1996).
} 
l'épreuve de sa justification dans la société. En observant les rythmes urbains de ces hommes, c'est-à-dire la manière dont ceux-ci habitent et «font » la ville en devenant eux-mêmes des représentations masculines en mouvement, on accède à une nouvelle forme d'urbanité masculine. La figure de «l'homme urbain » s'impose alors sous plusieurs angles: d'abord comme une autre déclinaison possible des masculinités contemporaines tanzaniennes en vogue, ensuite comme une des composantes de cet « argile passionnelle que la ville façonne de diverses manières pour donner forme aux structures locales de pouvoir» (De Boeck \& Plissart, Ibid. : 241), enfin comme une de ces catégories sociales « en train de se faire».

\section{Conclusion}

La mise en perspective de l'instabilité de la ville - dans sa définition comme dans son développement - avec l'exemple de certaines trajectoires et figures urbaines également touchées par l'incertitude a permis de faire une percée dans le quotidien de ces hommes célibataires tanzaniens, et de mesurer l'ampleur de leurs défis statutaires. Tout en proposant une lecture réaliste et à petite échelle de ce qui «fait» la ville, des liens ont été tracés entre le fait de «se faire habitant dans la ville » et «se faire acteur de la construction de celle-ci », et de mettre en relief les façons dont l'incertitude se greffe dans l'urbanisme de la ville et, par répercussion, dans l'urbanité de ses habitants. Le retour sur l'habitant et sur l'analyse de sa vie quotidienne nous a donc paru indispensable pour que soit justifiée l'existence de la ville au-delà de son architecture.

L'incertitude a été abordée sous l'angle des domesticités célibataires, masculines de surcroît, qui imposent des formes constantes, et quotidiennes, de négociation de l'identité genrée et sociale. Dans ce cas, le foyer masculin célibataire a été considéré non pas comme un lieu de repli exclusif et objectivé, mais comme un dispositif permettant de réguler la vie urbaine au quotidien. La gestion de cette incertitude en marche dans la ville a par ailleurs montré comment elle contribuait à façonner pour ces hommes les modalités de leur urbanité. Dans la société tanzanienne, l'homme seul est en passe de devenir, avec ses fragilités, ses hésitations et ses contradictions, un nouvel acteur «moderne » de la ville est-africaine en mutations. Bien que la ville se définisse par une avancée de l'incertitude au quotidien, la voie de l'auto-affirmation semble néanmoins transparaître pour ses habitants en situation de fragilité. L'exemple de l'homme seul décline l'image à son paroxysme au sein de ces nouvelles mutations et nouveaux paysages. Il permet de voir comment l'individu est touché à la fois dans sa quotidienneté, dans sa matérialité, dans son urbanité et jusque dans sa masculinité. Vécue avant tout comme une contrainte, l'incertitude peut dans ce cas être considérée par défaut comme un terrain d'entente, un compromis, qui donne à la ville comme à ses habitants les moyens de s'installer progressivement dans ce qui bouge et ce qui change.

En somme, cette définition sociale du développement urbain, qui s'intéresse aux métamorphoses et aux incertitudes qui sont présentes dans les façons de vivre la ville, invite à regarder la ville est-africaine moderne sous un autre angle, non pas comme une chose visible ou une totalité à saisir, mais comme un tout décomposé, un hologramme perceptible, " appréhensible » et vécu en situation (Agier, 2009: 6). 


\section{BIBLIOGRAPHIE}

Agier M., 2009, Esquisses d'une anthropologie de la ville : lieux, situations, mouvements, LouvainLa-Neuve : Academia-Bruylant.

Banegas R. \& Warnier J.-P., 2001, « Nouvelles figures de la réussite et du pouvoir », Politique Africaine 82 (numéro spécial 'Figures de la réussite et imaginaires politiques').

Boeck (de) F. \& Plissart M.-F., 2005, Kinshasa, Récits de la ville invisible, Tervuren : Musée Royal de l'Afrique Centrale.

Bonnin Ph. \& Villanova (de) R. (dir.), 1996, D’une maison l'autre. Parcours et mobilités résidentielles, Paris : Créaphis.

Connell R.W., 2000, «Masculinités et mondialisation », in Nouvelles approches des hommes et $d u$ masculin, Welzer-Lang D (dir.), Toulouse: Presses Universitaires du Mirail, pp.195-219.

—2005 (2ème édition) Masculinities, UK, Polity Press.

Fouéré M.-A., 2009, «J. K. Nyerere entre mythe et histoire : analyse de la production d'une mémoire publique officielle en Tanzanie post-socialiste », Les Cabiers d'Afrique de l'Est, n41, pp. 197-224.

-2011, "Tanzanie: L'imaginaire national à l'épreuve du postsocialisme », Politique Africaine, 121, pp. 69-85.

Haram L., 2009, «Uncertain Livelihood: Women Challenging the hegemonies of Male Sexual Dominance ", in Dealing with Uncertainty in Contemporary African Lives, Haram L. \& Bawa Yamba C., Stockholm : Nordiska Afrikainstitutet, pp.194-212.

Mbembe A., 2010, Sortir de la grande nuit. Essai sur l'Afrique décolonisée, Paris : La Découverte.

Meyer B. \& Geschiere P., 1999, Globalization and Identity: Dialectics of Flow and Closure, Oxford : Blackwell.

Mugambi H. N. \& Allan T.J., 2010, Masculinities in African Literary and Cultural Texts, UK, Ayebia.

Silberschmidt M., 2005, "Poverty, Male Disempowerment, and Male Sexuality: Rethinking Men and Masculinities in Rural and Urban East Africa ", in African Masculinities. Men in Africa from the Late Nineteenth Century to the Present, Ouzgane L. \& Morrell R., USA, Palgrave Macmillan, pp. 189-203.

Sunseri Th., 2002, 'Vilimani'. Labor Migration and Rural Change in Early Colonial Tanzania, UK, James Currey Ltd.

Weiss B., 2009, Street Dreams and Hip Hop Barbershops. Global Fantasy in Urban Tanzania, Indianapolis : Indiana University Press.

Welzer-Lang D. \& Zaouche Gaudron C., 2011, Masculinités : état des lieux, Toulouse : Erès. 